\title{
Global Controllability of Hybrid Systems with Controlled and Autonomous Switchings ${ }^{\star}$
}

\author{
Ekaterina S. Lemch ${ }^{1}$, Shankar Sastry ${ }^{1}$, and Peter E. Caines ${ }^{2}$ \\ 1 Dept. of Electrical Engineering and Computer Sciences \\ 275 Cory Hall 1774 \\ University of California at Berkeley \\ Berkeley, CA, 94720-1774, U.S.A. \\ $\{$ lemch, sastry\}@eecs. berkeley.edu \\ 2 Department of Electrical and Computer Engineering \\ McGill University \\ 3480 University Street, Montreal \\ Quebec, Canada H3A 2A7 \\ peterc@cim.mcgill.ca
}

\begin{abstract}
In this paper we investigate the question of the global controllability posed for control hybrid systems with autounomous and controlled swithchings. The main tool for our analysis is the notion of the controlled hybrifold. New sufficient conditions for the global controllability are obtained in terms of the so-called hybrid fountains.
\end{abstract}

\section{Introduction}

In this paper we consider systems which have a hybrid nature, in the sense that the dynamics of the system combines continuous and discrete components. We model control hybrid systems as a tuple consisting of a state space, a set of admissible continuous and discrete controls, a family of controlled vector fields assigned to each discrete state, a collection of autonomous and controlled switching surfaces, and a collection of the correspondint reset maps.

The main question investigated in the paper is the controllability of control hybrid systems. This issue has been addressed in 1511213. In particular, in [12], the notion of controllability for hybrid systems is formalized by continuity of system functions. In [1], the authors derive a necessary and sufficient algebraic condition for a certain subclass of piecewise affine hybrid systems. In [13], a sufficient condition for controllability of hybrid systems is formulated in terms of the so-called arrival sets.

Because of the complexity of the problem of the global controllability, its unlikely to find uniform sufficient conditions for general hybrid systems. Thus, we restrict our study to a special subclass of control hybrid systems, namely, the systems that can be represented as hybrifolds. The notion of the hybrifold

\footnotetext{
* The work is supported by DARPA under F33615-98-C-3614 and NSERC grant number OGP 0001329.
} 
was originally introduced in [14] and extended to control hybrid systems with autonomous switchings in 9] (see also [6], where the hybrifold notion is used in problems of optimal control for hybrid systems). In this paper we generalize the results formulated in [9] to systems that admit both autonomous and controlled switchings. New sufficient conditions for the global controllability are obtained in terms of the so called hybrid fountains. The advantage of the approach proposed in this paper is in the fact, that the fountain property can be verified at each particular state and, hence, there is no need to invoke a dynamic programminglike procedure to determine arrival sets of the system.

The paper is organized as follows. In Section 2, we formally define the class of control hybrid systems $H$ under our consideration and specify the standard assumptions on the continuous and discrete parts of the dynamics of $H$. In Section 3, we generalize the notion of the hybrifold to control hybrid systems with controlled and autonomous switchings and define a controlled flow on the hybrifold. Section 4 relates the global controllability of $H$ to the global controllability of the associated controlled hybrifold. In Section [5, we introduce the notion of a hybrid fountain and provide new sufficient conditions for the global controllability of control hybrid systems.

\section{Regular Control Hybrid Systems: Standing Assumptions}

We consider control hybrid systems which in this paper are taken to be of the following form.

Definition 1. An $n$-dimensional control hybrid systems $H$ is a 6 -tuple

$$
H=\{Q, \mathcal{D}, \mathcal{S}, \mathcal{R}, \Sigma, \mathcal{F}\}
$$

where

$Q=\{1, \cdots, k\}, 1 \leq k<\infty$, is a set of discrete states (which are called control locations);

$\mathcal{D}=\left\{D_{i} ; i \in Q, D_{i} \subset \mathbb{R}^{n}\right\}$ is a collection of domains of $H$;

$\mathcal{S}=\mathcal{S}_{a} \cup \mathcal{S}_{c}$ is a collection of autonomous and controlled switching surfaces;

$\mathcal{R}=\mathcal{R}_{a} \cup \mathcal{R}_{c}$ is a collection of autonomous and control resets.

$\Sigma=\Sigma_{c} \cup \Sigma_{d}$ is the set of admissible continuous and discrete controls;

$\mathcal{F}=\left\{f_{i} ; i \in Q, f_{i}: D_{i} \times \mathbb{R}^{n_{u}} \rightarrow \mathbb{R}^{n}\right\}$ is a collection of control vector fields assigned to each location;

Each of these components shall be further specified in the next part of the section.

The collections of autonomous swithching surfaces (called guards) and autonomous resets

$$
\mathcal{S}_{a}=\left\{S_{a}^{i j} ; \quad(i, j) \epsilon E_{a}\right\} \quad \mathcal{R}_{a}=\left\{R_{a}^{i j} ; \quad(i, j) \epsilon E_{a}\right\}
$$


where $E_{a} \subset Q \times Q$, are such that each guard $S_{a}^{i j}$ is a subset of $D_{i}$ and each autonomous reset $R_{a}^{i j}$ is a continuous injective map acting from $S_{a}^{i j}$ to $D_{j}$.

Similarly, for controlled switching surfaces and resets we have:

$$
\mathcal{S}_{c}=\left\{S_{c}^{i j} ; \quad(i, j) \epsilon E_{c}\right\} \quad \mathcal{R}_{c}=\left\{R_{c}^{i j} ; \quad(i, j) \epsilon E_{c}\right\}
$$

where $E_{c} \subset Q \times Q$, each controlled switching surface $S_{c}^{i j}$ is a subset of $D_{i}$, and each controlled reset $R_{c}^{i j}$ is a continuous injective map acting from $S_{c}^{i j}$ to $D_{j}$.

The set of discrete controls $\Sigma_{d}$ is taken to be $\left\{\sigma_{i j} ;(i, j) \in E_{c}\right\}$, where each $\sigma_{i j}$ is a discrete control that can be applied at (and only at) states $x \epsilon S_{c}^{i j}$.

Take an arbitrary initial state $\left(i, x_{0}\right)$ which does not lie on any of the switching surfaces. Then, for any control $u \epsilon \Sigma_{c}$, the systems evolves according to the ODE

$$
\dot{x}=f_{i}(x, u), \quad x(0)=x_{0}
$$

until it hits (at some point $\bar{x}$ ) either (i) a guard $S_{a}^{i j}$ or (ii) a controlled switching surface $S_{c}^{i k}$.

In the former case (i), the system necessarily switches to the discrete location $j$ and the continuous component of the states resets to $R_{a}^{i j}(\bar{x})$. Next, the system evolves according to the dynamics $f_{j}$ in the domain $D_{j}$.

In the latter case (ii), we distinguish two possibilities.

(ii.a) The discrete control $\sigma_{i k}$ is applied at $\bar{x}$; then the system switches to the location $k$ and the continuous component of the state resets to $R_{c}^{i k}(\bar{x})$. Next, the system evolves according to $f_{k}$ in $D_{k}$.

(ii.b) The discrete control $\sigma_{i k}$ is not applied; the system continues evolving according to $f_{i}$ in $D_{i}$.

The following definition of a hybrid time trajectory is based on [10 11].

\section{Definition 2 (Forward Hybrid Time Trajectory).}

A (forward) hybrid time trajectory is a sequence of semi-closed intervals

$$
\tau=\left\{\left[\tau_{i}, \tau_{i+1}\right) ; 1 \leq i \leq N \leq \infty, \tau_{i}<\tau_{i+1}\right\}
$$

We shall use the symbol $N(\tau)$ to denote the size of the time trajectory (i.e. the number of semi-intervals in the sequence $\tau$ ), the symbol $\langle\tau\rangle$ to denote the set $\{1,2, \cdots, N(\tau)\}$, and the symbol $\tau_{\infty}$ to denote the execution time, which, for a finite $N(\tau)$, is defined to be $\tau_{\infty} \underline{\Delta} \tau_{N(\tau)+1}-\tau_{1}$.

Based on the above description of the evolution of $H$, for any control pair $(u, \sigma)$, where $u$ is a continuous control in $\Sigma_{c}$ and $\sigma$ is a sequence of discrete controls $\left\{v_{1}, v_{2}, \cdots, v_{k} ; \quad v_{i} \in \Sigma_{d}\right\}$, we can define the notion of the control execution $\chi=\{\tau, q, \phi\}$ of $H$ starting at the initial state $p \in D$, where

(i) $\quad \tau$ is a hybrid time trajectory that contains the sequence of the switching times;

(ii) $q:\langle\tau\rangle \rightarrow Q$ is a map that contains the sequence of discrete locations visited by the hybrid trajectory; 
(iii) $\phi=\left\{\phi_{j} ; j \epsilon\langle\tau\rangle\right\}$ is the collection of continuously differentiable maps of $t$ that satisfies the corresponding ODEs and the switching conditions as described above.

As in [14, we shall restrict ourselves to the study of hybrid systems that are subject to the following assumptions.

A1 The control hybrid system $H_{w}$ is deterministic and non-blocking, for any control pair $w=(u, \sigma)$.

A2 For each $i \in Q, D_{i}$ is assumed to be a non-empty, closed, contractible $n$ dimensional sub-manifold of $\mathbb{R}^{n}$, with a piecewise smooth boundary.

A3 For each $e \epsilon E_{a}$ and $\tilde{e} \epsilon E_{c}$, the guard $S_{a}^{e}$ and the controlled switching surface $S_{c}^{\tilde{e}}$ are closed $(n-1)$-dimensional submanifolds with a piecewise smooth boundary. These sets have finite number of connected components.

A4 All resets maps are continuous and injective.

A5 None of the autonomous transition sets (i.e. $\left\{S_{a}^{e}, R_{a}^{e}\left(S_{a}^{e}\right) ; \quad\right.$ e $\left.\epsilon E_{a}\right\}$; denoted ATrans) have intersections with the controlled transition sets (i.e. $\left\{S_{c}^{\tilde{e}}, R_{c}^{\tilde{e}}\left(S_{c}^{\tilde{e}}\right) ; \tilde{e} \in E_{c}\right\}$; denoted $C T$ rans). Further, for any two (autonomous or controlled) transition sets $B_{1}, B_{2}$ (denoted Trans), we have

$$
B_{1} \cap B_{2} \neq \emptyset \Rightarrow B_{1}=S_{c}^{i j_{1}}=B_{2}=S_{c}^{i j_{2}}
$$

for some $i, j_{1}, j_{2} \in Q$.

Remark 1. We note that the restriction $S_{a}^{e} \cap S_{c}^{\tilde{e}}=\emptyset$ comes from the fact that $H$ is assumed to be deterministic. The rest of the restrictions of $A 5$ can be somewhat relaxed. We impose $A 5$ to avoid cumbersome technical details, while illustrating the point that certain hybrid systems can be represented as manifolds (termed hybrifolds), and thus, results on the global controllability formulated for manifolds can be transformed to hybrid systems.

Next we list the assumptions on the continuous part of the dynamics of $H$.

B1 For each $i \in Q, X_{i} \in C^{r}\left(D_{i} \times U ; \mathbb{R}^{n}\right), r \in\{1,2, \cdots, \infty, \omega\}$, where $C^{\omega}$ denotes the class of analytic functions.

B2 The set of admissible control functions

$$
\Sigma_{c}=\Sigma_{c}{ }^{s}\left(\mathbb{R} ; \mathbb{R}^{n_{u}}\right), s \in\{1,2, \cdots, \infty\}
$$

is the set of all $\mathbb{R}^{n_{u}}$-valued bounded piecewise $C^{s}\left(\mathbb{R} ; \mathbb{R}^{n_{u}}\right)$ functions of time with limits from the right. Hence any $u \in \Sigma_{c}$, defined on some $\left[T_{1}, T_{2}\right)$, $T_{2}<\infty$, is $C^{s}$ on $\left[T_{1}, T_{2}\right)$ with the exception of a finite number of points.

For the results formulated in this paper we shall need $r=1, s=1$.

Definition 3. A control hybrid system satisfying assumptions A1-A5 and B1B2 is called a regular control hybrid system with controlled and autonomous switchings. 
Finally, it shall be assumed that the system $H$ is non-Zeno in the sense that in finite time only a finite number of discrete transitions may be generated.

Lemma 1. Let $H$ be a regular control hybrid system. For any control pair $(u, \sigma)$ and any $p \in D$, there exists a unique control execution of $H$ starting at $p$.

\section{Controlled Hybrifold}

In [14], a set $M_{H}$ (called the hybrifold) is constructed from a hybrid system with autonomous switchings $H$. In this section we generalize this procedure to hybrid systems with autonomous and controlled switchings, prove that the resulting set $M_{H}$ is a manifold and, finally, define the controlled hybrid flow on $M_{H}$.

The basic idea in the construction of the hybrifold is to glue together each switching surface to the image of the corresponding reset map by identifying any state $p \epsilon S_{s}^{e}$, where $e \epsilon E_{s}, s=a, c$, with the corresponding image $R_{s}^{e}(p)$. So an $\|Q\|$

equivalence relation $\sim$ on $D \Delta \bigcup_{i=1} D_{i}$ is generated by

$$
p \sim R_{s}^{e}(p)
$$

for all $e \epsilon E_{s}$ and $p \epsilon S_{s}^{e}$. This relation gives rise to the quotient space

$$
M_{H}=D / \sim
$$

where each equivalence class is collapsed to a point.

Let $\pi$ be the natural projection map

$$
\pi: D \rightarrow M_{H}
$$

which assigns to each $p$ its equivalence class. We put the quotient topology on $M_{H}$, i.e. the smallest topology in which $V \subset M_{H}$ is open if and only if $\pi^{-1}(V) \subset D$ is open (in the relative topology of $D$ ).

Definition 4. The set $M_{H}$ with the quotient topology defined on it is called the controlled hybrifold associated with $H$.

The following result is based on [14].

Theorem 1. $M_{H}$ is a topological $n$-manifold with boundary.

Henceforth we shall deal not with the original domains $D_{i}$ but rather with the hybrifold $M_{H}$. We shall assume, without loss of generality, that $M_{H}$ is embedded in $\mathbb{R}^{m}$, for some $n \leq m<\infty$.

Definition 5 (Hybrid Control Flow). Take an arbitrary continuous control $u \in \Sigma_{c}$ defined on some $\left[T_{1}, T_{2}\right), T_{2}<\infty$, a sequence of discrete controls $\sigma$, and a state $x \in M_{H}$. Let $p \in \pi^{-1}(x)$. 
As follows from Lemma 1, there exists a unique control execution $\chi=$ $\{\tau, q, \phi\}$ of $H$ starting at $p$ which corresponds to the control pair $(u, \sigma)$.

We shall use the symbol $\Psi^{H}(t, x, u, \sigma), t \in\left[T_{1}, T_{2}\right)$, to denote the controlled hybrid flow on $M_{H} \cdot \Psi^{H}(t, x, u, \sigma)$ is defined as follows:

$$
\Psi^{H}(t, x, u, \sigma) \underline{\Delta}\left(\phi_{i}(t)\right), \text { for any } i \epsilon\langle\tau\rangle \text { and } t \epsilon\left[\tau_{i}, \tau_{i+1}\right) .
$$

In particular, we have $\Psi^{H}\left(\tau_{1}, x, u, \sigma\right)=\pi\left(\phi_{1}\left(\tau_{1}\right)\right)=\pi(p)=x$.

Remark 2. We note that, as follows from the Assumption A5, the definition of the control flow on $M_{H}$ does not depend on the choice of the representative $p$ in the equivalence class $x$.

Lemma 2. For any control $u$, the controlled hybrid flow $\Psi^{H}(\cdot, x, u, \sigma)$ is continuous on $M_{H}$ with respect to the argument $t$.

Proof: This follows from the fact that all points of discontinuity of the control hybrid execution are removed by identifying them with their images under the corresponding reset maps.

\section{The Global Controllability of Hybrid Systems}

Let $H$ be an arbitrary regular control hybrid system and $M_{H}$ its controlled hybrifold. In this section we relate the global controllability of the total domain $D$ of $H$ with the global controllability of $M_{H}$.

Definition 6 (Accessible sets of the control hybrid system $H$ ). Let $p \in D$. We shall say that a state $p^{\prime} \in D$ is accessible from $p$ (with respect to $V \subset D)$ if there exists a continuous control $u \in \Sigma_{c}$, defined on some $\left[T_{1}, T_{2}\right)$, $T_{2}<\infty$, and a sequence of discrete controls $\sigma=\left\{v_{1}, \cdots, v_{k}\right\}$ such that the corresponding control execution $\chi=(\tau, q, \phi)$ of $H$ starting at $p$ satisfies

(i) $\phi_{N(\tau)}(T)=p^{\prime}$, for some $T \epsilon\left[\tau_{N(\tau)} ; \tau_{N(\tau)+1}\right)$; and

(ii for any $j \epsilon\langle\tau\rangle$ and $t \epsilon\left[\tau_{j} ; \tau_{j+1}\right), \phi_{j}(t) \epsilon V$.

The set of all states in $D$ accessible from $p$ (with respect to $V$ ) shall be denoted by $A_{D}^{V}(p)$. In the case $V=D$, we shall write $A_{D}(p)$.

Thus we assumed that an accessible state $p^{\prime}$ can be reached from $p$ in finite time using a finite number of switching (or jumps) between control locations.

Remark 3. We observe that, as follows from the definition of the control execution of $H, R_{s}^{e}(p) \epsilon A_{D}(p)$, for any state $p \epsilon S_{s}^{e}, e \epsilon E_{s}, s=a, c$.

Similarly, we can define the accessible states using the dynamics of the controlled hybrifold $M_{H}$. 


\section{Definition 7 (Accessible sets of the controlled hybrifold $M_{H}$ ).}

Let $x \in M_{H} \subset \mathbb{R}^{m}$. We shall say that a state $x^{\prime} \in M_{H}$ is accessible from $x$ (with respect to $V \subset M_{H}$ ) if there exists a continuous control $u \epsilon \Sigma_{c}$ defined on some $\left[T_{1}, T_{2}\right), T_{2}<\infty$, and a sequence of discrete controls $\sigma=\left\{v_{1}, \cdots, v_{k}\right\}$ such that

(i) $x^{\prime}=\Psi^{H}(T, x, u, \sigma)$, for some $T \epsilon\left[T_{1}, T_{2}\right)$; and

(ii) for any $T_{1} \leq t \leq T, \Psi^{H}(t, x, u, \sigma) \epsilon V$.

The set of all states in $M_{H}$ accessible from $x$ (with respect to $V$ ) shall be denoted by $A^{V}(x)$. In the case $V=M_{H}$, we shall write $A(x)$.

The set of all states co-accessible to $p$ (to $x$ ), with respect to $V \subset D$ (with respect to $V \subset M_{H}$ ), in $H$ (in $M_{H}$ ) is defined dually and shall be denoted as $C A_{D}^{V}(p)$ (as $C A^{W}(x)$ ).

Remark 4. We observe that for any $p \in D$ and any neighborhood $V$ of $p$ in $D$, we have

$$
\pi\left(A_{D}^{V}(p)\right) \subset A^{\pi(V)}(\pi(p)),
$$

where $\pi: D \longrightarrow M_{H}$ is the natural projection map. This is because any orbit in $D$ is projected by $\pi$ onto an orbit in $M_{H}$.

On the other hand, let $p, p^{\prime} \in D$ and let $\pi\left(p^{\prime}\right) \in A^{V}(\pi(p))$. Then there exist some $y, y^{\prime} \in D$ such that (i) $p \sim y, p^{\prime} \sim y^{\prime}$ and (ii) $y^{\prime} \in A_{D}^{\pi^{-1}(V)}(y)$. In other words, the existence of a trajectory from $\pi(p)$ to $\pi\left(p^{\prime}\right)$ in $M_{H}$ does not necessarily imply the existence of a control execution connecting $p$ to $p^{\prime}$; it only implies the existence of a control execution from some $y \in D$ to some $y^{\prime} \in D$, where $y \sim p$ and $y^{\prime} \sim p^{\prime}$.

This is particularly easy to see in the situation, where at some controlled switching surface $S_{c}^{i j}$ at least two discrete controlled $\sigma_{i j_{1}}, \sigma_{i j_{2}}$ can be applied. Take $x \epsilon S_{c}^{i j}$ and consider $y_{1}=R_{c}^{i j_{1}}(x)$ and $y_{2}=R_{c}^{i j_{2}}(x)$. Then $x, y_{1}, y_{2}$ lie in the same equivalence class (they are glued together in $\left.M_{H}\right)$ and, hence, $\pi\left(y_{1}\right)$ and $\pi\left(y_{2}\right)$ are mutually accessible in $M_{H}$. At the same time $y_{1}$ and $y_{2}$ are not necessarily mutually accessible in $D$.

Hence in general, we do not have the reverse to (2) inclusion and we can only guarantee that for any $x \in M_{H}$ and $V \subset M_{H}$,

$$
A^{V}(x) \subset \pi\left\{\bigcup_{p \in \pi^{-1}(x)} A_{D}^{\pi^{-1}(V)}(p)\right\} .
$$

Definition 8. We say that a set $D_{1} \subset D$ is controllable with respect to $D_{2} \subset D$ for the control hybrid system $H$ if $A_{D}^{D_{2}}(p)=D_{1}$, for all $p \epsilon D_{1}$.

In the particular case when $D_{1}=D, D_{2}=D$, and $A_{D}(p)=D$, for all $p \epsilon D$, we shall say that the total domain $D$ is globally controllable for $H$.

Similarly, we shall say that a set $C_{1} \subset M_{H}$ is controllable with respect to $C_{2} \subset$ $M_{H}$ if $A^{C_{2}}(x)=C_{1}$, for all $x \in C_{1} . M_{H}$ is globally controllable if $A(x)=M_{H}$, for all $x \in M_{H}$. 
Theorem 2. Let $H$ be a regular control hybrid system. Then the total domain $D$ is globally controllable if and only if the associated hybrifold $M_{H}$ is globally controllable.

\section{Proof:}

$\Longrightarrow$ Let $D$ be globally controllable. Then, using Remark 4 (2), we obtain for any $x \in M_{H}$,

$$
M_{H}=\pi(D)=\pi\left(A_{D}(p)\right) \subset A^{\pi(D)}(\pi(p))=A(x) \subset M_{H},
$$

where $p$ is an arbitrary point in the set $\pi^{-1}(x) \subset D$. Hence $A(x)=M_{H}$, for any $x \in M_{H}$, and $M_{H}$ is globally controllable.

$\Longleftarrow$ Conversely, let $M_{H}$ be globally controllable. Take any $p, p^{\prime} \in D$. Each of them could lie in any of the sets

$$
\text { CTrans, ATrans, } \tilde{D} \triangleq D-\text { Trans, }
$$

i.e. there are 9 possible cases.

Consider, for instance, the case when $p \in R_{c}^{e}\left(S_{c}^{e}\right)$ and $p^{\prime} \epsilon R_{c}^{e^{\prime}}\left(S_{c}^{e^{\prime}}\right)$, for some $e=(i, j), e^{\prime}=\left(i, j^{\prime}\right) \in E_{c}$. Take the inverse image $y^{\prime}=\left\{R_{c}^{e^{\prime}}\right\}^{-1}\left(p^{\prime}\right)$. As follows from the description of the hybrid executions given in Section [2, there exist states $z \in D_{j} \cap \tilde{D}$ and $z^{\prime} \epsilon D_{i} \cap \tilde{D}$ such that $z$ is accessible from $y$ and $z^{\prime}$ is co-accessible to $y^{\prime}$. Next note, that since $z, z^{\prime} \in D-$ Trans and $\pi$ is 1 to 1 on $\tilde{D}$, from the existence of an orbit connecting $\pi(z)$ to $\pi\left(z^{\prime}\right)$ in $M_{H}$ follows the existence of a control execution that drives $z$ to $z^{\prime}$. Finally, combining all the accessibility relations for $p, z, z^{\prime}, y^{\prime}, p^{\prime}$ we conclude that $p^{\prime} \in A_{D}(p)$.

The rest of the cases can be considered in an analogous manner. Thus $A_{D}(p)=D$, for any $p \epsilon D$, and $D$ is globally controllable.

The above result allows us to use the hybrifold and the continuous controlled hybrid flow defined on it in order to study the global controllability of the original control hybrid system. The advantage of this approach is in the fact that the controllability results formulated for differential control systems acting on subsets or sub-manifolds of $\mathbb{R}^{n}$ can be transformed to control hybrid systems. This shall be demonstrated in the next section.

\section{$5 \quad$ Hybrid Fountains}

In this section we introduce the notion of a hybrid fountain which we shall use as the main hypothesis in our controllability result. Henceforth the symbol $B_{\delta}(x)$, where $x \in M_{H}, 0<\delta \in \mathbb{R}^{1}$, shall denote the $m$-dimensional ball with the center $x$ and the radius $\delta$. The sets $A^{B_{\delta}(p)}(p)$ and $C A^{B_{\delta}(p)}(p)$ shall be denoted as $A^{\delta}(p)$ and $C A^{\delta}(p)$, respectively.

Definition 9. A state $x \in M_{H}$ is called a hybrid fountain if

$$
\begin{gathered}
\exists \mu>0 \forall \delta, 0<\delta<\mu, A^{\delta}(x)-\{x\} \text { and } C A^{\delta}(x)-\{x\} \\
\text { are non-empty, open sets. }
\end{gathered}
$$


If the function $\rho \triangleq \sup \{\mu$; such that the condition (4) holds $\}$ is continuous at $x$, we shall say that $x$ is a continuous hybrid fountain. If $\rho$ is unbounded at $x$ we consider it to be continuous at $x$.

The reader is referred to [2,3,7] for applications of the fountain condition to the study of ordinary differential systems acting on subsets of $\mathbb{R}^{n}$. See also [8, where a set of algebraic conditions for verification of the fountain property is presented, and [4] where applications to hierarchical hybrid control theory are outlined.

Henceforth we shall use the term controlled closed orbit in the sense of controlled loop.

Theorem 3. Let each $x \in M_{H}$ be a continuous hybrid fountain and let for each $x \in M_{H}$ there exist a control $u \in \Sigma_{c}$ such that $x$ lies on a nontrivial (controlled under $u$ ) closed orbit in $M_{H}$. Then each connected component of $\left[M_{H}\right]^{\circ}$ is controllable with respect to $M_{H}$.

Proof: Let $C$ denote one of (the finite number of) the connected components of $\left[M_{H}\right]^{\circ}$. For any two states $x, x^{\prime}$ in $C$ we define a relation $\sim_{o}$ in such a way that $x \sim_{o} x^{\prime}$ if and only if there exists a (controlled) nontrivial closed orbit in $M_{H}$ passing through both $x$ and $x^{\prime}$, i.e. there exists a control pair $u, \sigma$ defined on some $\left[T_{1}, T_{2}\right), T_{2}<\infty$, such that

(i) $\exists T, T_{1}<T<T_{2}, \quad \Psi\left(T_{1}, x, u, \sigma\right)=\Psi(T, x, u, \sigma)$; and

(ii) $\exists \bar{t}, T_{1}<\bar{t} \leq T, \quad \Psi(\bar{t}, p, u, \sigma)=p^{\prime}$.

Clearly, the relation $\sim_{o}$ is reflexive (since each state in $M_{H}$ lies on a nontrivial orbit), symmetric and transitive. Hence there exists a partition of $C$ on the equivalence classes of $\sim_{o}$. Let $[x]$, for an arbitrary $x \in C$, denote the equivalence class containing $x$. We claim that $[x]$ is an open subset in $C$.

Indeed, take any $z \epsilon[x]$. Let $u$ and $0 \leq t<\infty$ be such that $z=\Psi(t, x, u, \sigma)$. Define $a=\Psi(t-\Delta, x, u, \sigma)$ and $b=\Psi(t+\Delta, x, u, \sigma), \Delta>0$. Then, since $a$ and $b$ are hybrid fountains, the sets $A^{\delta}(a)-\{a\}$ and $C A^{\delta}(b)-\{b\}$ are open, for sufficiently small $\delta>0$. Choose $\Delta$ so small that $z \in A^{\delta}(a)$ and $z \in C A^{\delta}(b)$ (this is possible since $a, b$ are continuous hybrid fountains). Then there exists an open neighborhood $N(z)$ of $z$ which lie in the intersection $\left(A^{\delta}(a)-\{a\}\right) \cap\left(C A^{\delta}(b)-\right.$ $\{b\})$. Each state $z^{\prime} \in N(z)$ is accessible from $a$ and co-accessible to $b$. Moreover, since $a, b \epsilon[x]$, we conclude that $z^{\prime}$ lies on a non-trivial orbit passing through $x$. This is true for all $z^{\prime} \in N(z)$, hence $N(z) \subset[x]$ and $[x]$ is open, as claimed.

For any $x, x^{\prime} \in C$ we have $[x] \cap\left[x^{\prime}\right] \neq \emptyset \Longrightarrow[x]=\left[x^{\prime}\right]$, so any two equivalence classes are either disjoint or coincide. Thus the set $C$ can be represented as the disjoint union $C=A \cup B$, where $A \underline{\Delta}[x]$, for some $x \in C$, and $B \Delta \bigcup_{\substack{x^{\prime} \in C \\ x^{\prime} \in\{x]}}\left[x^{\prime}\right]$.

$A$ and $B$ are open and disjoint. Since $C$ is connected, we conclude that $B$ is empty, i.e. any $x^{\prime} \in C$ is such that $x \sim_{o} x^{\prime}$. In other words, any two states in $C$ lie on a nontrivial controlled orbit in $M_{H}$ and hence, $C$ is controllable with respect to $M_{H}$. 
Remark 5. We note at this point that weaker recurrence conditions can be used instead of the existence of closed orbits. Also, for the proof of the above result, the continuous hybrid fountain condition (4) can be relaxed to

$\rho(x) \triangleq \sup \left\{\mu>0 ; A^{\mu}(x)-\{x\}, C A^{\mu}(x)-\{x\}\right.$ are non-empty, open sets $\}$ is continuous, for all $x \in M_{H}$.

Theorem 4. Assume that the hybrifold $M_{H}$ is connected and the conditions of Theorem 3 are satisfied. Then $M_{H}$ is globally controllable.

Proof: As has been shown in [14, $M_{H}$ is $n$-dimensional manifold (possibly with boundary). This implies, by definition, that for any boundary state in $\partial M_{H}$ there exists a neighborhood which is homeomorphic to $\mathbb{R}_{+}^{n}$. Hence $\left[M_{H}\right]^{\circ}$ and $M_{H}$ have the same number of connected components; in particular, $\left[M_{H}\right]^{\circ}$ is connected if and only if $M_{H}$ is connected.

Take any boundary state $x \in \partial M_{H}$. Then, since $x$ is a hybrid fountain, the sets $A^{\delta}(x)-\{x\}$ and $C A^{\delta}(x)-\{x\}$ are non-empty and open, for sufficiently small $\delta>0$. Hence there exist $a \epsilon\left(A^{\delta}(x)-\{x\}\right) \cap\left[M_{H}\right]^{\circ}$ and $b \epsilon\left(C A^{\delta}(x)-\{x\}\right) \cap\left[M_{H}\right]^{\circ}$.

For any state $p^{\prime} \epsilon\left[M_{H}\right]^{\circ}$ we can find a control $u \epsilon \Sigma_{c}$ which would drive $a$ $p^{\prime}$ and a control $u^{\prime} \epsilon \Sigma_{c}$ which would drive $p^{\prime}$ to $b$. This is because $a, b, p^{\prime}$ lie in $\left[M_{H}\right]^{\circ}$ and, as follows from Theorem 3, $\left[M_{H}\right]^{\circ}$ is controllable. We conclude that arbitrary $p \epsilon \partial M_{H}$ and $p^{\prime} \epsilon\left[M_{H}\right]^{\circ}$, and thus arbitrary $p, p^{\prime} \epsilon M_{H}$, are mutually accessible. Hence $M_{H}$ is globally controllable.

Consider the directed graph $\Gamma$ of $H$ which has vertices $Q$ and edges $E$. We can treat it as a finite state machine, by defining the transition function $\Phi: Q \rightarrow Q$ in such a way that for any $i, j \in Q, \Phi(i)=j$ if and only if $(i, j) \epsilon E$ or $i=j$.

Theorem 5. Assume that the conditions of Theorem 3 are satisfied. Then $M_{H}$ is globally controllable if and only if the graph $\Gamma=\{Q, E\}$ is controllable as a finite state machine.

\section{Proof:}

$\Longrightarrow$ Assume that $M_{H}$ is globally controllable. Then for any $i, j \in Q, i \neq j$, take some states $p \in D_{i}$ and $p^{\prime} \in D_{j}$. There exists a trajectory $\psi$ from $p$ to $p^{\prime}$ in $M_{H}$. Let the sequence $i=r_{1}, r_{2}, \cdots, r_{\ell}=j, \ell>1$, be such that $\psi$ switches consecutively from the domain $D_{r_{s}}$ to the domain $D_{r_{s+1}}$, where $s=1,2, \cdots, \ell-1$, using the corresponding guards and the images of the reset maps. Hence each consecutive pair $\left(r_{s}, r_{s+1}\right)$ belongs to $E$ and hence, there exists a trajectory from the state $i$ to the state $j$ in the graph $\Gamma$. Since this holds for an arbitrary pair $(i, j) \in Q$, we conclude that $\Gamma$ is controllable as a finite state machine.

$\Longleftarrow$ Conversely, assume that $\Gamma$ is controllable as a finite state machine. Then for any two states $p, p^{\prime} \epsilon D$ take $i$ and $j$ such that $p \epsilon D_{i}$ and $p^{\prime} \epsilon D_{j}$. If $i \neq j$, find a trajectory $i=r_{1}, r_{2}, \cdots, r_{\ell}=j, \ell>1$, in the graph $\Gamma$. Since each consecutive pair $\left(r_{s}, r_{s+1}\right)$ belongs to $E$, there exists a guard $G_{\left(r_{s}, r_{s+1}\right)}$ in the domain $D_{r_{s}}$ which is identified with the image of the reset map $R_{\left(r_{s}, r_{s+1}\right)}$ in the domain $D_{r_{s+1}}$. Hence the domains $D_{r_{s}}$ and $D_{r_{s+1}}$, and thus $D_{i}$ and $D_{j}$, lie in 
one connected component of $M_{H}$. This can be shown for all $i, j \in Q$. Hence $M_{H}$ is connected and, as follows from Theorem $4 M_{H}$ is globally controllable.

An application of the obtained results can be illustrated on a two water tank system example, which, for the lack of space, shall be described briefly. The water can be added to the system at some rate $w>0$ (where we treat the parameter $w$ as control) in two different modes:

1: the water is added (exclusively) via tank 1 ;

2: the water is added (exclusively) via tank 2 .

In addition to that, the water is removed from tank $i, i=1,2$, at some constant rate $v_{i}>0$. The two tank system can be modeled as a control hybrid system in the following way. We shall distinguish two control locations - each corresponds to one of the modes, i.e. $Q=\{1,2\}$. The continuous dynamics at the locations are as:

$$
\begin{aligned}
& q=1:\left\{\begin{array}{l}
\dot{x}=w-v_{1} \\
\dot{y}=-v_{2}
\end{array}(x, y) \in D_{1} \Delta\left\{\left[l_{1}, \infty\right) \times\left[l_{2}, \infty\right)\right\},\right. \\
& q=2:\left\{\begin{array}{l}
\dot{x}=-v_{1} \\
\dot{y}=w-v_{2}
\end{array}(x, y) \in D_{2} \triangleq\left\{\left[l_{1}, \infty\right) \times\left[l_{2}, \infty\right)\right\},\right.
\end{aligned}
$$

where $x, y$ denote the levels of water in the tanks 1 and 2 , respectively. The class of control functions is taken to be the set of all functions taking values in $\Re$ and satisfying $B_{2}$.

The guards are defined as

$$
G_{(1,2)}=1 \times\left\{(x, y) \epsilon D_{1} ; y=l_{2}\right\}, \quad G_{(2,1)}=2 \times\left\{(x, y) \epsilon D_{2} ; x=l_{1}\right\}
$$

The resets are defined in such a way that when hitting a guard in one domain the system switches to the other control location, without changing the continuous part of the state, i.e.

$$
R_{(1,2)}\left(1 ; x, l_{2}\right)=\left(2 ; x, l_{2}\right), \quad R_{(2,1)}\left(2 ; l_{1}, y\right)=\left(1 ; l_{1}, y\right)
$$

Furthermore, assume that for some level $y=l, l \geq l_{2}$, in the first tank, a discrete switching to the second tank is allowed.

To construct the corresponding controlled hybrifold we identify (via the identity reset maps) the $x=l_{1}, y=l_{2}, y=l$ axes of $D_{1}$ with the $x=l_{1}, y=l_{2}, y=l$ axes of $D_{2}$, respectively.

Using the obtained results, it can be verified that each state of the hybrifold is a hybrid fountain lying on a closed orbit. Hence, the two water tank system can be shown to be globally controllable.

Remark 6. In conclusion we note that algebraic conditions for verification of the fountain property at each state $x \in M_{H}$ shall be presented in a future version of the paper. 


\section{References}

1. A.Bemporad, G. Ferrari-Trecate, and R. Morari. Observability and controllability of piecewise affine and hybrid systems. In Proceedings of the 38th IEEE Control Systems Society Conference on Decision and Control, pages 3966-3971, Phoenix, AZ., Phoenix, AZ, 1999.

2. P.E. Caines and E.S. Lemch. On the global controllability of Hamiltonian and other nonlinear systems: fountains and recurrence. In Proceedings of the 37th IEEE Control Systems Society Conference on Decision and Control, pages 35753580, Tampa, FL, 1998.

3. P.E. Caines and E.S. Lemch. On the global controllability of nonlinear systems: fountains, recurrence, and application to Hamiltonian systems. Submitted to SIAM J. on Control and Optimization, 2000.

4. P.E. Caines and E.S. Lemch. Hierarchical hybrid systems: geometry, controllability and applications to air traffic control. Proceedings of 14th World IFAC, volume E, Beijing, China, July, 1999.

5. J. Ezzine and A.H. Haddad. Controllability and observability of hybrid systems. Int. J. Control, 49(6):2045-2055, 1989.

6. Ekaterina S. Lemch, Jianghai Hu, and Shankar Sastry. Approximation techniques in optimal control problems for a class of hybrid systems. Hybrid Systems: Computation and Control, Third International Workshop, 2001.

7. E.S. Lemch. Nonlinear and hierarchical hybrid control systems. Ph.D. thesis, McGill University, Canada, August, 1999.

8. E.S. Lemch and P.E. Caines. On the global controllability of nonlinear systems: algebraic conditions for the existence of fountains. Fourteenth International Symposium on Mathematical Theory of Networks and Systems, June, 19-23, 2000.

9. E.S. Lemch, S. Sastry, and P.E. Caines. On the global controllability of hybrid systems: Hybrifolds and fountains. In To Appear in Proceedings of the 39th IEEE Control Systems Society Conference on Decision and Control, Sydney, Australia, Sydney, Australia, 2000.

10. J. Lygeros, K.H. Johansson, S. Sastry, and M. Egerstedt. On the existence of executions of hybrid automata. In Proceedings of the 38th IEEE Control Systems Society Conference on Decision and Control. Phoenix, AZ, Phoenix, AZ, 1999.

11. J. Lygeros, C. Tomlin, and S. Sastry. Controllers for reachability specifications for hybrid systems. Automatica, 35(3), March, 1999.

12. A. Nerode and W. Kohn. Models for hybrid systems: automata topologies, controllability, observability. Hybrid Systems, pages 317-356, Berlin, Germany, 10-12 October, 1991.

13. J.H. Schuppen. A sufficient condition for controllability of a class of hybrid systems. Hybrid Systems: Computation and Control, First International Workshop, Berkeley, CA, April, 1998.

14. S.N. Simić, K.H. Johansson, S. Sastry, and J. Lygeros. Towards a geometric theory of hybrid systems. Hybrid Systems: Computation and Control, Third International Workshop, Pittsburgh, PA, 2000. 